\title{
The Liver Plays an Important Role in the Regulation of Somatostatin-14 in the Rat
}

\author{
Steven E. Raper, MD, Piyush C. Kothary, MS, Norihiro Kokudo, MD, John DelValle, MD, \\ Frederic E. Eckhauser, MD, Ann Arbor, Michigan
}

Since little is known about the in vivo disposition of circulating somatostatin-14 (SRIF-14), we examined hepatic processing of SRIF-14 in the rat. Three minutes after the intraportal injection of iodine $125\left({ }^{125} I\right)$-labeled SRIF-14, $16.0 \pm 2.0 \%$ of the injected dose is localized to the liver. In the presence of unlabeled SRIF-14, hepatic uptake can be decreased by $68 \%$. Five minutes after the intraportal injection of ${ }^{125}$ I-SRIF-14, $9.5 \pm 1.4 \%$ of the tracer is localized to the liver, more than any other organ tested. Serial collections of bile reveal peak radioactivity at between 10 and 20 minutes. Simultaneous administration of unlabeled SRIF-14 decreases biliary radioactivity by $40 \%$. HPLC analysis of radioactive bile reveals a chromatographic profile similar to that of intact SRIF and is $73 \%$ immunoprecipitable by an anti-SRIF antibody. Pretreatment with chloroquine, a lysosomal enzyme inhibitor, does not significantly decrease biliary radioactivity. We conclude that the data are consistent with saturable hepatic uptake and predominantly nonlysosomal transcellular transport.

$\mathrm{T}$ he liver appears to be an important determinant of circulating concentrations of various gastrointestinal peptides in the blood. Somatostatin-14 (somatotropin release-inhibiting factor or SRIF-14) is a tetradecapeptide first isolated in sheep hypothalamus and named for its ability to inhibit pituitary growth hormone secretion [1]. Peripheral blood concentrations of somatostatin-like immunoreactivity (SLI) are much lower than SLI concentrations in portal venous blood [2]. Clearance of SRIF-14 in an isolated, perfused rat liver model has been documented, and similar results have been obtained in pri-

From the Departments of Surgery (SER, PCK, NK, FEE) and Internal Medicine (JDV), University of Michigan Medical Center, Ann Arbor, Michigan.

Dr. Raper is the recipient of the Society for Surgery of the Alimentary Tract Career Development Award and Grant DK 42485 from the United States Public Health Service, Bethesda, Maryland.

Requests for reprints should be addressed to Steven E. Raper, MD, 2922H Taubman Health Care Center, 1500 E. Medical Center Drive, Ann Arbor, Michigan 48109-0331.

Presented at the 31st Annual Meeting of the Society for Surgery of the Alimentary Tract, San Antonio, Texas, May 15-16, 1990. mary cultures of isolated rat hepatocytes $[3,4]$. Nonspecific degradation at the plasma membrane has been believed to be primarily responsible for SRIF metabolic regulation, but specific binding, uptake, and intracellular processing have not been systematically studied.

Mammalian liver can bind certain peptide hormones and other circulating plasma proteins and process them by one of two pathways: (1) a direct pathway in which the peptides are transported intact into bile, or (2) an indirect pathway in which membrane vesicles fuse with lysosomes, are degraded, and are excreted into bile [5]. Such specific binding, uptake, and intracellular processing is called receptor-mediated endocytosis. Hepatic uptake and biliary secretion of at least two peptide hormones with significant effects on hepatic physiology-insulin and epidermal growth factor (EGF)-involve binding to well-characterized receptors [6,7]. Specific hepatic processing of cholecystokinin octapeptide (CCK-8) has also been documented, although the exact mechanism has not been determined $[8]$.

SRIF-14 has also been shown to alter certain aspects of hepatic physiology. In dogs, a continuous infusion of SRIF-14 decreases bile volume and bile acid-independent canalicular bile flow [9]. Bile acid-independent canalicular bile flow decreased in response to SRIF-14 in the intact rat as well as in the isolated perfused rat liver, again suggesting a direct effect of SRIF-14 on the liver [10]. In humans, a continuous intravenous infusion of SRIF-14 decreases bile secretion and the output of bile acids, cholesterol, and phospholipid [11].

Evidence from several laboratories suggests that SRIF-14 may affect the liver by inhibiting synthesis of the intracellular second messenger, cyclic AMP. Glucagon is believed to act via receptor-mediated stimulation of adenyl cyclase and the elevation of intracellular levels of cyclic AMP. Cyclic AMP production by rat liver slices decreases significantly in response to SRIF-14, in both the basal and glucagon-stimulated state [12]. SRIF-14 infusion inhibits glucagon-induced glucose release from the isolated perfused rat liver [13]. The glucagon-stimulated elevation of cyclic AMP in isolated hepatocytes can be blocked by SRIF [14].

Given the evidence for hepatic metabolism of SRIF14 and the documented effects of SRIF-14 on the liver, the purpose of the experiments described herein was as follows: (1) to study the hepatic uptake and disposition of SRIF-14 using an intact rat model and a highly purified preparation of radiolabeled SRIF-14 as a tracer; (2) to determine the presence of radioactivity in bile; (3) to characterize the form of biliary radioactivity; (4) to determine the role of lysosomal degradation in hepatic processing of SRIF-14. 


\section{MATERIAL AND METHODS}

Chemicals: Tyrosine 11 [tyr ${ }^{11}$ ]-SRIF-14 and SRIF14 were obtained from Peninsula Laboratories (Belmont, CA). Sodium iodide I $125\left({ }^{125} \mathrm{NaI}\right)$ was obtained from Amersham Corp (Arlington Heights, IL). Chloroquine and all other chemicals and reagents were obtained from Sigma Chemical Co. (St Louis, MO). Antibody Ab 8093 used for the immunoprecipitation studies was kindly supplied by Dr T Yamada.

Radioiodination of highly purified SRIF-14: [tyr ${ }^{11}$ ]-SRIF-14 was obtained commercially and radioiodinated by the chloramine-T method [15]. Briefly, ${ }^{125} \mathrm{NaI}(0.5 \mathrm{mCi})$, and chloramine- $\mathrm{T}(25 \mathrm{nmol})$ were incubated in $0.04 \mathrm{M}$ sodium phosphate buffer ( $\mathrm{pH} 7.4$ ) for 15 seconds. Sodium metabisulfite $(35 \mathrm{nmol})$ was used to stop the reaction. The radioligand was purified by reverse-phase high-performance liquid chromatography (HPLC) as described herein. Percent incorporation of radioactivity was assessed with an anti-SRIF antibody and found to be routinely greater than $90 \%$.

Surgical and anesthetic techniques: Male SpragueDawley rats, weighing 250 to $325 \mathrm{~g}$, were anesthetized as previously described [16]. Briefly, isoflurane/oxygen anesthesia induction is performed in a restraining chamber and subsequently administered by mask. After midline laparotomy, bile duct cannulation is performed 0.5 to 1.0 $\mathrm{cm}$ from the confluence of the hepatic ducts with PE-50 tubing using $2.5 \times$ surgical magnification. Intraportal injections are performed with 26-gauge needles, and hemostasis is achieved with thrombin-soaked pledgets.

HPLC analysis of ${ }^{125}$ I-[tyr $\left.{ }^{11}\right]$-SRIF-14: The HPLC runs are performed on a $\mu$ Bondapak $\mathrm{C}_{18}$ column $(0.39 \times$ $30 \mathrm{~cm}$, Waters Associates, Milford, MA) [17]. Briefly, the buffers used for elution are $2 \%$ trifluoroacetic acid (TFA) (Buffer A) and 50\% acetonitrile in 2\% TFA (Buffer $B)$. The columns are eluted at a flow rate of $1 \mathrm{~mL} /$ minute at room temperature using the following protocol: 5 minutes at $0 \% \mathrm{~B}, 17.5$ minutes gradient of $0 \%$ to $70 \% \mathrm{~B}$, 10 minutes at $70 \% \mathrm{~B}$, and 5 minutes at $70 \%$ to $0 \% \mathrm{~B}$. Eluted fractions are collected and counted for ${ }^{125} \mathbf{I}$.

\begin{tabular}{|c|c|}
\hline \multicolumn{2}{|c|}{$\begin{array}{l}\text { TABI.E I } \\
\text { Competition of SRIF-14 and }{ }^{125} \text { I-SRIF-14 for Hepatic } \\
\text { Uptake }(n=6)\end{array}$} \\
\hline & $\begin{array}{c}\text { CPM/g Liver }(\times 10-5) \\
(\text { mean } \pm \text { SEM })\end{array}$ \\
\hline 125|-SRIF-14 Alone & $2.87 \pm 0.32^{*}$ \\
\hline 125 I-SRIF-14 + SRIF-14 & $0.92 \pm 0.18$ \\
\hline
\end{tabular}

Immunoprecipitation: Ten microliters of $95 \%$ ethanol is added to $100 \mu \mathrm{l}$ of bile and centrifuged for 30 minutes at $4^{\circ} \mathrm{C}$ and 2,500 revolutions per minute to precipitate bile salts [18]. To the supernatant is added 300 $\mu \mathrm{L}$ of buffer and $100 \mu \mathrm{L}$ of a 1:10,000 dilution of antibody 8093 , a polyclonal rabbit anti-SRIF-14 antibody. Nonspecific binding is determined by using preimmune rabbit serum. After incubation overnight at $4^{\circ} \mathrm{C}$, activated charcoal is added and radioactivity in the pellet is counted. Results are expressed as percent total minus percent nonspecific radioactivity-bound.

Statistical analysis: Statistical analysis was done on a Macintosh II personal computer using the Statview II statistical software package (Abacus Concepts, Inc., Berkeley, CA). Analysis of variance with repeated measures and Student's unpaired $t$ test were used to compare data sets. Significance is assumed for a $p$ value less than or equal to 0.05 .

\section{RESULTS}

Hepatic uptake of 125I-SRIF-14: At 3 minutes after the intraportal injection of ${ }^{125}$ I-SRIF-14, $16.0 \pm 2.0 \%$ of the injected tracer was taken up by the liver. Saturability of SRIF uptake was demonstrated in the presence of a 500-fold excess of unlabeled SRIF-14 with only $5.1 \pm$ $1.0 \%$ taken up by the liver, a $68 \%$ decrease (Table I). Analysis of ${ }^{125}$ I-SRIF uptake revealed that liver and bile take up proportionately more radioactivity than would be

\begin{tabular}{|c|c|c|c|c|c|c|}
\hline \multicolumn{7}{|c|}{ TABLE II } \\
\hline \multirow[b]{2}{*}{ Organ } & \multicolumn{2}{|c|}{5 Minutes } & \multicolumn{2}{|c|}{30 Minutes } & \multicolumn{2}{|c|}{60 Minutes } \\
\hline & $\mathrm{CPM} / \mathrm{g}(\times 10-4)$ & $\begin{array}{c}\% \text { Injected } \\
\text { Dose }^{\dagger}\end{array}$ & $\mathrm{CPM} / \mathrm{g}(\times 10-4)$ & $\begin{array}{c}\% \text { Injected } \\
\text { Dose }^{\dagger}\end{array}$ & $\mathrm{CPM} / \mathrm{g}(\times 10-4)$ & $\begin{array}{c}\% \text { Injected } \\
\text { Dose }^{\dagger}\end{array}$ \\
\hline Bile & $21.9 \pm 2.7 \ddagger$ & $0.19 \pm 0.04$ & $36.2 \pm 14.0^{ \pm}$ & $0.88 \pm 0.06$ & $10.0 \pm 3.1 t$ & $3.88 \pm 1.05$ \\
\hline Liver & $9.9 \pm 1.4^{\ddagger}$ & $9.59 \pm 1.35$ & $6.0 \pm 0.2$ & $6.50 \pm 0.18$ & $2.0 \pm 0.5$ & $2.49 \pm 0.43$ \\
\hline Pancreas & $5.9 \pm 0.5$ & $0.43 \pm 0.03$ & $11.4 \pm 1.4^{\ddagger}$ & $0.72 \pm 0.04$ & $3.0 \pm 0.5$ & $0.26 \pm 0.08$ \\
\hline Gut & $3.7 \pm 0.4$ & $7.10 \pm 0.86$ & $4.4 \pm 1.1$ & $4.82 \pm 1.20$ & $2.5 \pm 0.5$ & $5.87 \pm 1.40$ \\
\hline Lungs & $5.6 \pm 0.3$ & $0.59 \pm 0.05$ & $6.1 \pm 1.0$ & $0.25 \pm 0.05$ & $2.5 \pm 0.5$ & $0.25 \pm 0.06$ \\
\hline Heart & $3.3 \pm 0.3$ & $0.29 \pm 0.01$ & $3.4 \pm 0.5$ & $0.24 \pm 0.01$ & $1.5 \pm 0.5$ & $0.15 \pm 0.03$ \\
\hline Spleen & $3.4 \pm 0.1$ & $0.24 \pm 0.02$ & $3.9 \pm 0.6$ & $0.20 \pm 0.03$ & $2.0 \pm 0.5$ & $0.11 \pm 0.02$ \\
\hline Kidney & $5.8 \pm 0.5$ & $0.63 \pm 0.12$ & $6.1 \pm 1.1$ & $1.09 \pm 0.12$ & $3.0 \pm 1.0$ & $0.90 \pm 0.29$ \\
\hline Blood & $7.4 \pm 0.3$ & ND & $9.9 \pm 1.1$ & ND & $3.5 \pm 0.5$ & ND \\
\hline
\end{tabular}




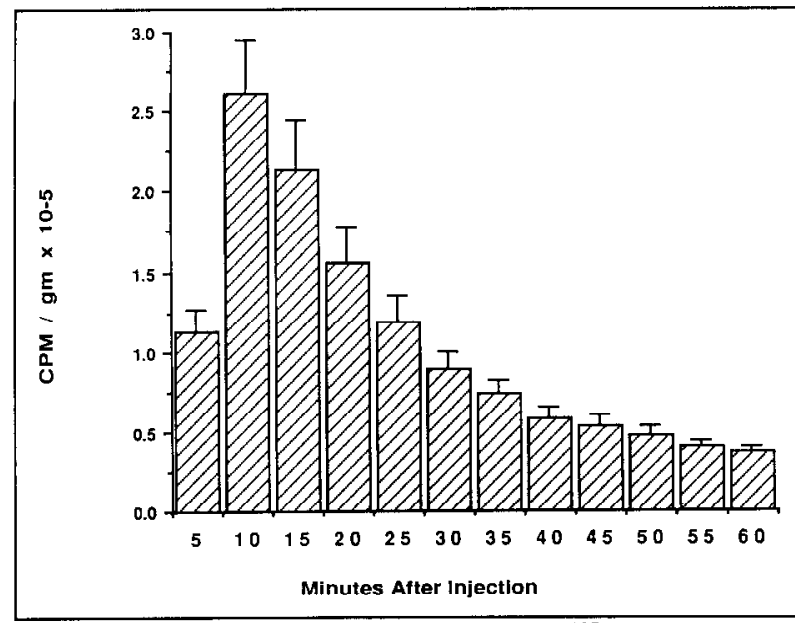

Figure 1. Time course of biliary secretion of ${ }^{125} /$-SRIF-14. Serial timed 5-minute collections of bile were obtained after the intraportal injection of a dose of ${ }^{125} \mid-S R I F-14\left(6 \times 10^{6} \mathrm{CPM}\right) .(n=4$; error bars $=$ SEM).

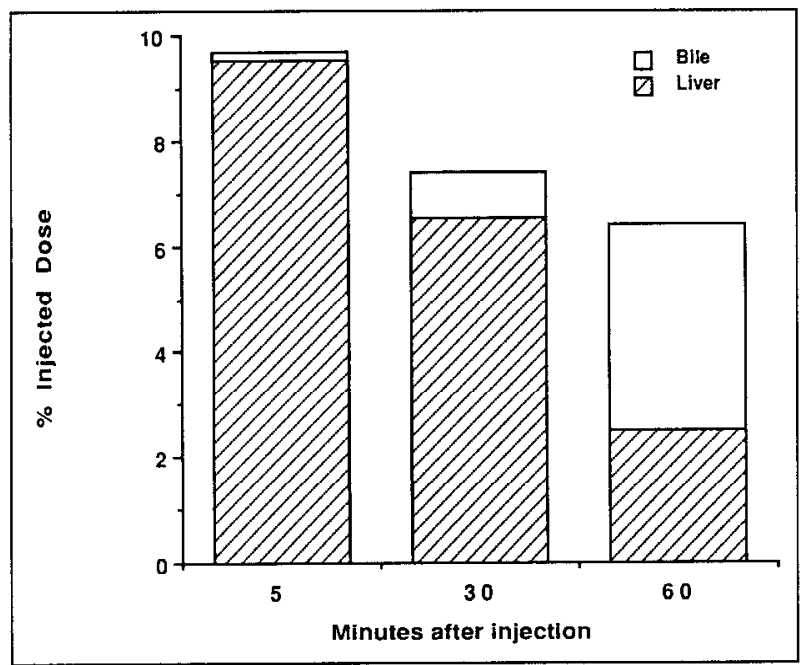

Figure 2. Ratio of biliary-to-hepatic radioactivity with time. The ratio of biliary and hepatic radioactivity at various times after injection is displayed as a stacked bar graph. The ordinate represents the percentage of an injected dose of ${ }^{125}$ |-SRIF-14 (6.2 $\times$ $10^{6} \mathrm{CPM}$ per rat; $n=4$ to 7 ).

expected on a weight basis when compared with other organs or biologic fluids. At 5 minutes after injection, hepatic uptake of ${ }^{125} \mathrm{I}$-SRIF was $9.88 \times 10^{4} \pm 1.44 \times 10^{4}$ counts per minute $(C P M) / g$ (mean \pm SEM), significantly higher $(p<0.05)$ than even that found in the pancreas, another organ known to express somatostatin receptors and to be physiologically regulated by SRIF (Table II). Bile contained $2.18 \times 10^{5} \pm 0.27 \times 10^{5} \mathrm{CPM} / \mathrm{g}$, threefold higher than blood (Table II).

If bile is collected for 30 or 60 minutes, respectively, before the organs are harvested and analyzed for radioactivity, the tissue differences in ${ }^{125}$ I-SRIF uptake are abolished (Table II). By 60 minutes, only biliary radioactivity remains significantly higher than would be expected on a weight basis. The data reflect the essentially complete degradation of ${ }^{125}$ I-SRIF-14 by 60 minutes after injection. Biliary radioactivity at 30 minutes can also be significantly decreased from $4.06 \times 10^{5} \pm 0.32 \times 10^{5} \mathrm{CPM} / \mathrm{g}$ bile to $2.51 \times 10^{5} \pm 1.08 \times 10^{5} \mathrm{CPM} / \mathrm{g}$ bile $(\mathrm{p}<0.05)$ by the simultaneous injection of a 500 -fold excess of unlabeled SRIF-14, again suggesting that the uptake of SRIF-14 is a saturable process.

Time course of biliary secretion of ${ }^{125}$ I-SRIF-14: The kinetics of appearance of radioactivity from ${ }^{125} \mathrm{I}$ SRIF-14 in bile were examined. After intraportal injection of ${ }^{125}$ I-SRIF-14, the appearance of radioactivity in bile increased rapidly, peaked at between 10 and 20 minutes, and gradually declined over the next 40 minutes (Figure 1). A comparison of the ratio of biliary to hepatic radioactivity at 5,30 , and 60 minutes revealed a progressive decline in the percentage of an injected dose found in the liver and a progressive increase in the percentage of biliary radioactivity (Figure 2 ).

Characterization of biliary ${ }^{125} I$ after intraportal injection of ${ }^{125} \mathrm{I}-\mathrm{SRIF}-14$ : To determine the nature of the radioactive label in bile after in vivo administration of ${ }^{125}$ I-SRIF-14, we examined bile containing ${ }^{125}$ I by HPLC and immunoprecipitation with a SRIF-specific antibody. Figure 3 shows results of analysis of radioactivity in bile using HPLC. Prior to HPLC analysis, the bile was first subjected to ethanol precipitation at $4^{\circ} \mathrm{C}$. The protocol used in analyzing biliary radioactivity was exactly the same as that used for analysis of the starting material. HPLC analysis of the bile reveals a peak of radioactivity that elutes at the same place as the starting material, although the peak is broad with respect to the starting tracer. Unlabeled bile "spiked" with ${ }^{125}$ I-SRIF-14 also exhibited a single peak of radioactivity, showing an elution pattern identical to the starting tracer. The elution peak for all three forms of radioactivity came at $27 \mathrm{~min}$ utes in the HPLC system described.

Immunoprecipitation of the HPLC peak of radioactivity with antibody 8093 , the same antiserum used to assess purity of the starting tracer, revealed that $73 \%$ of the counts were specifically bound by anti-SRIF antibody when compared with normal rat serum. These combined studies (HPLC and immunoprecipitation) suggest that after intraportal injection of ${ }^{125}$ I-SRIF-14, the SRIF that appears in bile is immunologically intact.

Effect of chloroquine on biliary secretion of ${ }^{125}$ I from SRIF-14: Chloroquine is a basic compound that inhibits lysosomal enzyme function by preventing lysosomal acidification. Lysosomotropic agents, such as chloroquine, are known to inhibit the hepatic transport and biliary excretion of a number of macromolecules. Figure 4 illustrates that chloroquine administration had no significant effect on the time course or quantity of biliary 125I-SRIF-14 secretion, although there was a trend toward less radioactivity at 10 and 20 minutes. The data suggest that lysosomes do not play a major role in the hepatic processing of SRIF.

\section{COMMENTS}

In these experiments, we have directly demonstrated the hepatic uptake and biliary secretion of SRIF-14. Our 
results demonstrate that (1) rat liver can rapidly and efficiently extract SRIF-14 from portal blood; (2) uptake is saturable; (3) uptake is followed by biliary secretion of radioactivity that appears identical to the starting label; (4) uptake and secretion are not significantly altered by chloroquine, a potent lysosomal enzyme inhibitor.

By use of the intact rat model, we have demonstrated organ-specific differences in the uptake of radiolabeled ${ }^{125}$ I-SRIF-14. Rat liver has significantly higher ${ }^{125} \mathrm{I}-$ SRIF-14 uptake than any other organ tested, including the pancreas and stomach, two organs whose functions are known to be physiologically regulated by this important peptide $[19,20]$. The percent uptake of an injected dose of ${ }^{125}$ I-SRIF is low compared with other biologically active peptides, such as epidermal growth factor (EGF) and transforming growth factor-beta (TGF- $\beta$ ). St Hiliare et al [21] were able to demonstrate a $95 \%$ hepatic uptake of a dose of intraportally injected EGF at 10 minutes, and Coffey et al [22] documented 53\% hepatic uptake of an intraportally injected dose of TGF- $\beta$ at 60 minutes. Efficient hepatic uptake and processing has also been documented for CCK-8, another small but biologically important gastrointestinal peptide. In one report, $79 \%$ of an intraportally injected dose of ${ }^{125} \mathrm{I}-\mathrm{CCK}-8$ was extracted by rat liver within a 20 -minute period, suggesting rapid, efficient uptake and processing [23]. Hepatic uptake and processing of pentagastrin have also been reported.

The lower percentage of uptake of ${ }^{125}$ I-SRIF-14 may relate to either technical differences in labeling of peptide or to the metabolism of SRIF-14 at other sites. A theoretical disadvantage of the chloramine-T method is the possibility of metabisulfite (used to stop the reaction) oxidation of the disulfide bond of the [tyr $\left.{ }^{11}\right]-S R I F-14$ molecule, which may in turn impair recognition by membrane-binding proteins. The chloramine-T technique has been used with success in a number of other experimental systems, including the stomach and isolated brain microvessels $[17,19]$. With respect to SRIF degradation in other organs, such as the nervous system, membranebound proteases may be responsible for rapid SRIF degradation. In the plasma, SRIF-14 has a half-life of about 3 minutes and appears to be degraded by endothelial cellassociated proteases throughout the central nervous system [17].

As mentioned, our results indicate that radioactive label taken up by the liver is rapidly transported into the bile. A labeled peptide could theoretically arrive in bile by paracellular or transcellular routes. A paracellular pathway seems unlikely because the process is partially blocked by the presence of unlabeled SRIF-14, suggesting saturation of a specific binding protein. Also, the concentration of label in bile is much greater than that in the bloodstream, as might be expected if an active secretion rather than a passive diffusion were occurring. Therefore, it seems likely that SRIF is transported by a transcellular pathway, presumably through the hepatocyte.

There are several transcellular pathways by which SRIF might be routed into bile. The nonspecific uptake of

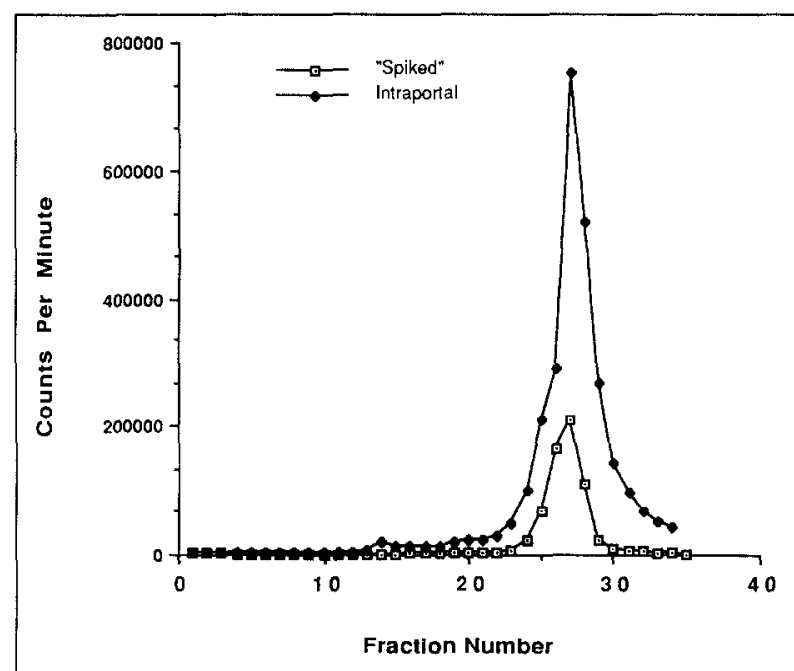

Flgure 3. HPLC elution profile of biliary ${ }^{125}$-SRIF-14. HPLC analysis of biliary radioactivity was performed under the same conditions as those for analysis of the original tracer. "Intraportal" represents biliary radioactivity from rats intraportally injected with ${ }^{125}$ I-SRIF-14. "Spiked" represents HPLC analysis of ${ }^{125}$-SRIF-14 added to unlabeled bile from control rats. The data are representative of five separate experiments.

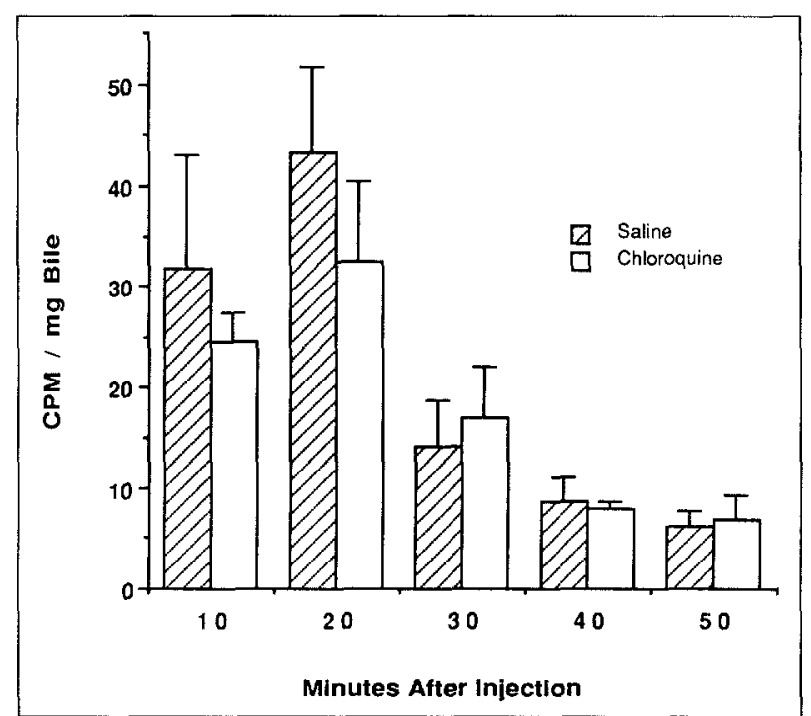

Figure 4. Effect of chioroquine on biliary secretion of ${ }^{125}$-SRIF14. Chloroquine $(50 \mathrm{mg} / \mathrm{kg})$ was administered intraperitoneally 2 and 1 hours before intraportal injection of ${ }^{125}$ I-SRIF-14 $\left(2 \times 10^{6}\right.$ CPM). Open bars represent rats given intraperitoneal $0.9 \%$ saline, hatched bars represent rats given intraperitoneal chloroquine. The data are expressed as $\mathrm{CPM} / \mathrm{g}$ bile (mean $\pm \mathrm{SEM} ; n=$ 8 to 12).

water, solute, and small molecules is called pinocytosis. Pinocytosis does not involve binding to specific receptors and should not be saturable by excess unlabeled peptide. Receptor-mediated endocytosis and vesicular transport of proteins into bile is another possibility. Two such vesicular pathways across the hepatocyte have been proposed: (1) a lysosomal, or indirect, pathway; or (2) a vesicular, or direct, pathway $[24,25]$. Biliary excretion of asialoglycoproteins occurs via the lysosomal pathway. Asialoglycoproteins are mammalian serum glycoproteins that have 
been altered to expose the penultimate galactose residue, a marker for senescence that in effect targets the protein for hepatic degradation [24]. In general, molecules processed through this pathway are excreted into bile in metabolized or degraded forms. This pathway is sensitive to lysosomotropic agents such as chloroquine, which inhibit lysosomal function by preventing intralysosomal acidification and inactivation of lysosomal acid proteases responsible for degradation of proteins, such as EGF. In our experiments, chloroquine did not significantly alter the appearance or time course of radioactivity into bile. These results may be taken as preliminary evidence against a role for hepatic lysosomes in the hepatic uptake and biliary secretion of SRIF.

In contrast, polymeric immunoglobulin $\mathrm{A}(\mathrm{pIgA})$ is taken up by the liver and secreted into bile via the direct pathway. Compounds such as PIgA that utilize hepatocyte vesicles are generally secreted into bile as intact molecules and appear in bile sooner than those excreted by lysosomes. In our experiments, the identification of radioactivity in bile that is similar to the starting material and that is largely immunologically recognizable suggests minimal degradation, as would be expected for the direct pathway. Furthermore, the kinetics of uptake of label into bile are more rapid than have been described for proteins that undergo lysosomal degradation. Although further experiments are clearly necessary, our findings can be interpreted as preliminary evidence for a vesicular, or direct, transport pathway for SRIF-14 in the liver.

The physiological purpose served by the rapid and efficient secretion of SRIF-14 into bile remains a matter of speculation. Since SRIF-14 is documented to have antiproliferative effects on the pancreas, it could conceivably be an antiproliferative factor for the biliary or even the intestinal epithelium [26]. SRIF-14 is known to regulate exocrine pancreatic and gastric secretion. It may also inhibit secretory functions of the biliary epithelium. Such a contention is strengthened by the recent identification of somatostatin-containing cells within the intra- and extrahepatic biliary tree [27]. Alternatively, the biliary secretion of SRIF-14 may provide a mechanism for regulating circulating somatostatin levels. Even if the major function of biliary secretion is to clear SRIF-14 from the portal blood, SRIF-14 could still exert a direct physiologic effect on the liver before secretion into bile.

In conclusion, our experimental results underscore the important and unique role of the liver in the metabolism of somatostatin. Not only does the liver remove a significant quantity of one of the naturally occurring forms of this hormone, SRIF-14, but it does so in an efficient and saturable manner. After hepatic uptake, the liver rapidly transports the hormone across the hepatocyte and secretes it into bile. Our initial experiments aimed at dissecting the details of this process are consistent with specific hepatic uptake and transcellular transport occurring predominantly in a nonlysosomal vesicular compartment.

\section{REFERENCES}

1. Brazeau P, Vale W, Burgus R, et al. Hypothalamic polypeptide that inhibits the secretion of immunoreactive pituitary growth hormone. Science 1973; 179: 77-9.
2. Patel YC, Wheatley T, Ning C. Multiple forms of immunoreactive somatostatin: comparison of distribution in neural and nonneural tissues and portal plasma in the rat. Endocrinology 1981; 109: 1943-9.

3. Sacks HS, Terry LC, Wright RK, Stentz FB. Somatostatin metabolism: differences in clearance of $\mathrm{N}$-terminal and central portions of molecule during perfusion of rat liver. Am J Physiol 1984; 246: G226-34.

4. Conlon JM, Whittaker J, Hammond V, Alberti KGMM. Metabolism of somatostatin and its analogues by the liver. Biochim Biophys Acta 1981; 677: 234-42.

5. Burwen SJ, Barker ME, Goldman IS, Hradek GT, Raper SE, Jones AL. Transport of epidermal growth factor by rat liver: evidence for a non-lysosomal pathway. J Cell Biol 1984; 99: 1259-65. 6. Pezzino V, Vigneri R, Cohen D, Goldfine ID. Regenerating rat liver: insulin and glucagon serum levels and receptor binding. Endocrinology 1981; 108: 2163-9.

7. Marti U, Burwen SJ, Jones AL. Biological effects of epidermal growth factor, with emphasis on the gastrointestinal tract and liver: an update. Hepatology 1989; 9: 126-38.

8. Gores GJ, LaRusso NF, Miller LJ. Hepatic processing of cholecystokinin peptides. I. Structural specificity and mechanism of hepatic extraction. Am J Physiol 1986; 250: G344-9.

9. Lewis MH, Baker AL, Moossa AR. Effect of somatostatin on determinants of bile flow in unanesthetized dogs. Ann Surg 1982; 195: 97-103.

10. Ricci GL, Fevery J. Cholestatic action of somatostatin in the rat: effect on the different fractions of bile secretion. Gastroenterology 1981; 81: 552-62.

11. Magnusson I, Einarsson K, Angelin B, Nyberg B, Bergstrom $\mathrm{K}$, Thulin $\mathrm{L}$. Effects of somatostatin on hepatic bile formation. Gastroenterology 1989; 96: 206-12.

12. Catalan RE, Avila C, Vila T, Castillon MP. Somatostatin effect on cyclic AMP levels mediated by glucagon stimulation in rat liver. Metabolism 1978; 27: 1359-60.

13. Sacks H, Waligora K, Matthews J, Pimstone BL. Inhibition by somatostatin of glucagon-induced glucose release from the isolated perfused rat liver. Endocrinology 1977; 101: 1751-9.

14. Oliver J, Long K, Wagle S, Allen DO. Somatostatin inhibition of glucagon-stimulated adenosine 3'-5' monophosphate accumulation in isolated hepatocytes. Proc Soc Exp Biol Med 1976; 153 $367-9$.

15. Hunter WM, Greenwood FC. Preparation of iodine-131 labelled human growth hormone of high specific activity. Nature 1962; 194: 495-6.

16. Raper SE, Barker ME, Burwen SJ, Jones AL. Isoflurane as an anesthetic for experimental animal surgery. Anat Rec 1987; 218: 116-22.

17. Pardridge WM, Eisenberg J, Yamada T. Rapid sequestration and degradation of somatostatin analogues by isolated brain microvessels. J Neurochem 1985; 44: 1178-84.

18. Kothary PC, Vinik AI, Owyang C, Fiddian-Green RG. Immunochemical studies of molecular heterogeneity of cholecystokinin in duodenal perfusate and plasma in humans. J Biol Chem 1983; 258: 2856-63.

19. Park J, Chiba T, Yamada T. Mechanisms for direct inhibition of canine gastric parietal cells by somatostatin. J Biol Chem 1987 262: $14190-6$.

20. Chariot J, Roze G, Vaille C, Debray C. Effccts of somatostatin on the external secretion of the pancreas in the rat. Gastroenterology $1978 ; 75: 832-7$

21. St Hilaire RJ, Hradek GT, Jones AL. Hepatic sequestration and biliary secretion of epidermal growth factor: Evidence for a high-capacity uptake system. Proc Natl Acad Sci (USA) 1983; 80: 3797-801.

22. Coffey RJ, Kost LJ, Lyons RM, Moses HL, LaRusso NF Hepatic processing of transforming growth factor $\beta$ in the rat: uptake, metabolism and biliary excretion. J Clin Invest 1987; 80: 750-7.

23. Gores GJ, Miller LJ, LaRusso NF. Hepatic processing of 
cholecystokinin peptides. II. Cellular metabolism, transport, and biliary excretion. Am J Physiol 1986; 250: G350-6.

24. Schwartz AL. The hepatic asialoglycoprotein receptor. Crit Rev Biochem Mol Biol 1983; 16: 207-33.

25. Jones AL, Renston RH, Burwen SJ. Uptake and intracellular disposition of plasma-derived proteins and apoproteins by hepatocytes. Prog Liver Dis 1983; 7: 51-69.

26. Morisset J. Somatostatin: a potential antigrowth factor for the exocrine pancreas. Regul Pept 1984; 10: 11-22.

27. Kurumaya H, Ohta G, Nakanuma Y. Endocrine cells in the intrahepatic biliary tree in normal livers and hepatolithiasis. Arch Pathol Lab Med 1988; 113: 143-7.

\section{DISCUSSION}

Keith Kelly (Rochester, MN): The fact that the molecule gets into the bile relatively unaltered suggests that perhaps it has some effect as it passes through the biliary tree either on the gallbladder or the sphincter of Oddi. Could you speculate on that?

Donald Kaminski (St. Louis, MO): Do you think there is an enterohepatic circulation of somatostatin with absorption of somatostatin from the intestinal tract? Secondly, can you speculate on the correct dose range for somatostatin for a variety of its therapeutic uses, such as inhibition of hormone secretion and treatment of intestinal fistulas?

Iawrence Way (San Francisco, CA): The fact that the molecule is passed through the liver intact suggests the possibility of tracking it in its intracellular transit. Have you been able to label it and examine it histologically in any way to see exactly where it is?

Steven E. Raper (closing): Dr. Kelly, there are several possibilities. Specific intracellular alterations may occur as a result of somatostatin binding to a membrane receptor. Signal transduction may then effect intrahepatic changes in physiology prior to secretion of somatostatin into the biliary tract. Once in the bile, somatostatin could interact with bile duct epithelium to alter ductular secretion. These hypotheses require further investigation.

Dr. Kaminski, in terms of enterohepatic circulation, it is difficult to know whether somatostatin could get from the upper duodenum all the way down to the distal ileum without some form of intraluminal degradation. Also, significant plasma degradation can occur, so that enterohepatic circulation is unlikely. As to your second question, commercially available forms of somatostatin analogues are administered at doses of 100 to $600 \mu \mathrm{g}$ daily in divided doses. For native somatostatin, physiologic effects have been seen with circulating levels in the nanomolar range. Given the presence of somatostatin-containing cells in many target organs, however, local concentrations of somatostatin may be much higher.

Dr. Way, autoradiographic studies are ongoing, but I don't have the pictures yet to confirm transhepatic transport. 